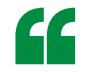

Thus, the

postsynaptic

function of

complexins

is mediated

via SNARE

complexes, but

does not involve

synaptotagmin 1
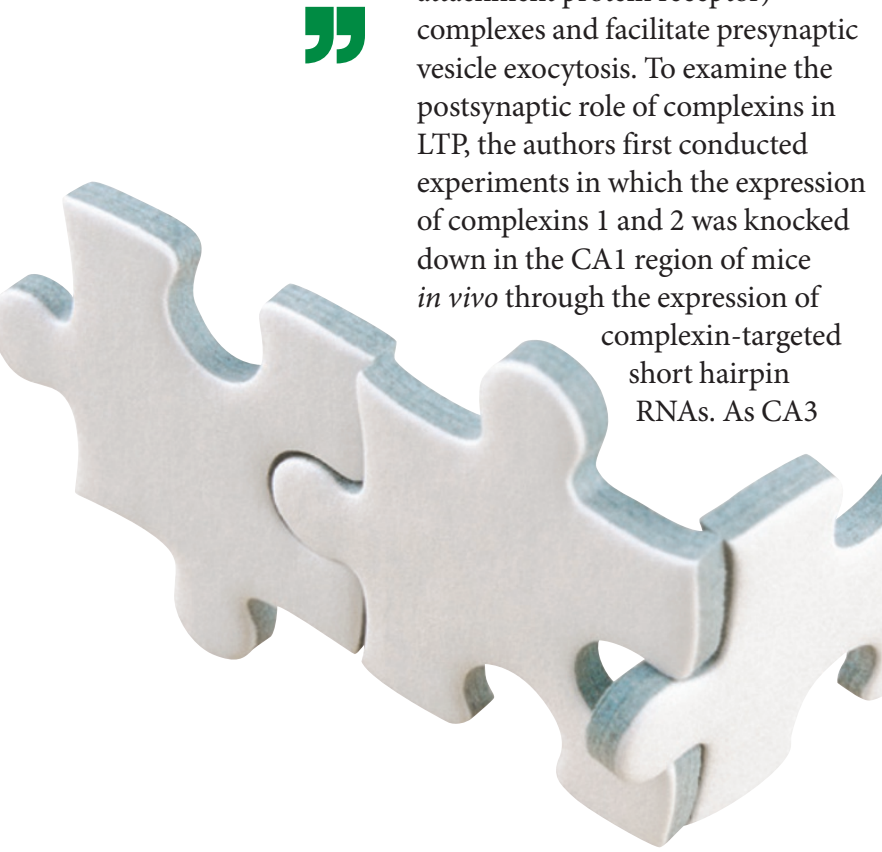

Long-term potentiation (LTP) may be a key process in learning and memory and, at the cellular level, involves the activation of NMDAtype glutamate receptors (NMDARs) at postsynaptic terminals, which leads to the calcium-dependent exocytosis of AMPA-type glutamate receptors (AMPARs). The identity of the molecules that trigger AMPAR exocytosis is unclear, but a new study shows that postsynaptic complexins might have an important role in this process.

Complexins comprise a family of small, highly charged cytosolic proteins that bind SNARE (soluble $N$-ethylmaleimide-sensitive-factor attachment protein receptor) complexes and facilitate presynaptic vesicle exocytosis. To examine the postsynaptic role of complexins in LTP, the authors first conducted experiments in which the expression of complexins 1 and 2 was knocked down in the CA1 region of mice in vivo through the expression of complexin-targeted short hairpin RNAs. As CA3

\title{
Adding a piece to the LTP jigsaw
}

pyramidal cells may form synapses onto CA1 pyramidal cells and LTP can be induced in these synapses in slice preparations, this approach allowed the postsynaptic function of complexins in LTP to be assessed.

In acute hippocampal slices prepared from these animals, LTP of CA3-CA1 synapses was impaired, although long-term depression and basal synaptic transmission were unaffected, indicating that postsynaptic complexins have a specific role in LTP.

The replacement of wild-type complexin in CA1 neurons with mutant forms of complexin that impaired SNARE binding also impaired LTP. However, knockdown of the expression of synaptotagmin 1 in CA1 neurons - a crucial component of the presynaptic release machinery that makes transmitter release calcium-dependent in hippocampal pyramidal cells - did not impair LTP of CA3-CA1 synapses. Thus, the postsynaptic function of complexins in LTP is mediated via SNARE complexes, but does not involve synaptotagmin 1 .

The authors switched to a hippocampal neuronal culture model of LTP, in which LTP was generated pharmacologically. As had been shown previously, LTP caused an increase in AMPAR plasma membrane expression and synaptic strength. Importantly, complexin knockdown prevented the NMDAR-triggered increase in AMPAR cell surface expression, and expression of the complexin mutants failed to rescue this effect, suggesting that complexins have a key role in AMPAR exocytosis.

The possibility that LTP impairment in neuronal cultures following complexin knockdown was due to a shift in the localization of the intracellular pool of AMPARs and not to a blockade of AMPAR exocytosis was excluded: the total levels of glutamate receptor 1 (GluA1)-containing AMPARs in dendrites were the same regardless of complexin knockdown, as was the proportion of recycling endosomes containing AMPARs. The constitutive endocytosis of endogenous surface AMPAR was also unaffected by complexin knockdown.

Together, these results establish complexins as key molecules in postsynaptic events during LTP. Nevertheless, as the authors acknowledge, more research is required to develop a level of understanding of the molecular mechanisms underlying LTP that is similar in depth to that of the mechanisms mediating neurotransmitter release.

Man Tsuey Tse

ORIGINAL RESEARCH PAPER Ahmad, M. et al. Postsynaptic complexin controls AMPA receptor exocytosis during LTP. Neuron 73, 260-267 (2012) 